\title{
Gonadal and Sexual Function in Young/Middle Aged Human immunodeficiency virus (HIV)-Infected Men
}

\author{
Santi $D^{1,2}$, Brigante $G^{1,2}$, Diazzi $C^{1,2}$, De Vincentis $S^{1,2}$, Zona $S^{3}$, Guaraldi $G^{3}$, Simoni ${ }^{1,2}$, Rochira $V^{1,2}$
}

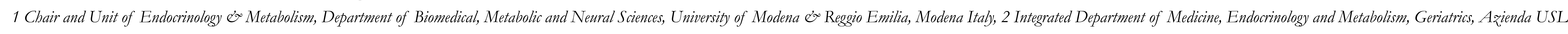
of Modena, NOCSAE of Baggiovara, Modena, Italy,3Metabolic Clinic, Infectious and Tropical Disease Unit, Department of Medical and Surgical Sciences for Cbildren \& Adults, University of Modena and Reggio E, Modena, Italy

\section{INTRODUCTION}

HIV infection is associated to an increased prevalence of agerelated comorbidities (1). Among these, erectile dysfunction (ED) and testosterone (T) deficiency are a common finding in HIV-infected men treated with highly active antiretroviral therapy (HAART) (2,3). However, the real prevalence of $T$ deficiency in the context of HIV-infection remains not well defined. The aim of the study is to investigate the sexual function by validated questionnaires such as International Index of Erectile function (IIFE)-15 and Structured Interview on Erectile Dysfunction (SIEDY), and the gonadal status by the gold standard isotopic dilution-liquid chromatography-tandem mass spectrometry (ID-LC-MS/MS), considered the gold standard in steroid hormones measurement (4), to better define prevalence of ED and T deficiency in HIV-infection.

\section{MATERIALS and METHODS}

We performed a multicenter, prospective, cross-sectional, observational study on 68 HIV-infected male patients (mean age=44years) with ongoing HAART, attending the Clinic of Infectious Diseases. IIEF-15 questionnaire was used to assess ED: a score $\leq 25$ at erectile domain was diagnostic of ED (5). Sexual function was studied also by SIEDY questionnaire, an easy tool for the identification of intra-psychic component (6). ID-LC-MS/MS was used for hormonal assays. Serum total $\mathrm{T}<300$ $\mathrm{ng} / \mathrm{dL}$ was suggestive for T deficiency (7).

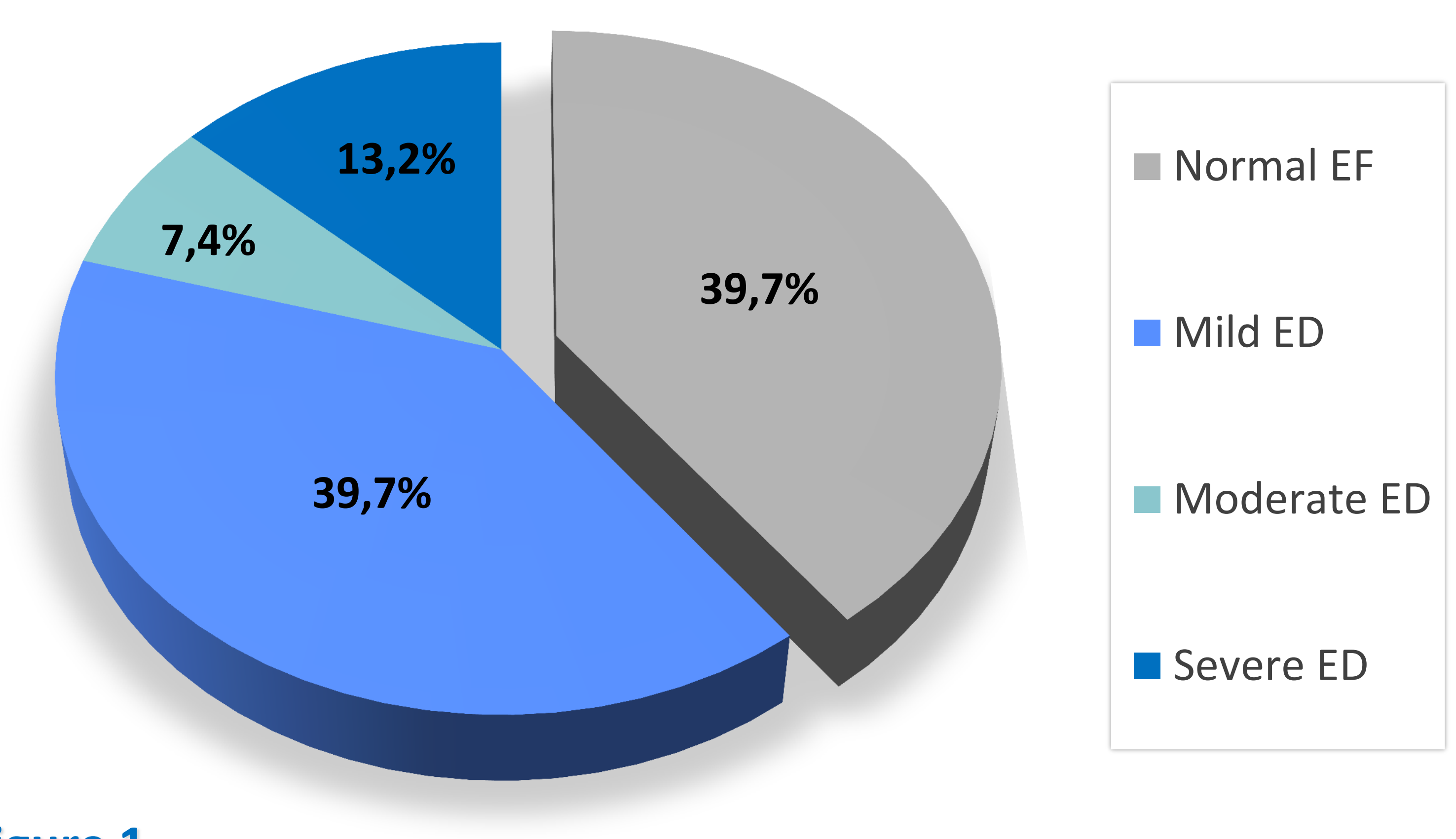

Figure 1.

Prevalence and characterization of ED, according to scores at EF domain of IIEF-15.

\section{RESULTS}

IIEF-15 erectile domain is impaired in $60.3 \%$ of patients, with a $13.2 \%$ of severe form (Fig.1). $11 \%$ of subjects declared the use of PDE5-inhibitors and they present higher scores at questionnaires. SIEDY organic scale scores were significantly impaired in patients with hypertension $(p=0.013)$ and Hepatitis $C$ virus (HCV) infection ( $p=0.007)$, whereas psychogenetic scale was impaired only in HCV-infected men $(p=0.008)$. T deficiency is found in $10 \%$ of subjects with a longer time of HIV-infection (Fig.2) and HAART. Comparing patients with or without T deficiency IIEF-15 and SIEDY scores are similar in both groups ( $p=0.134$ and $p=0.284$, respectively). However, serum $T$ levels were significantly lower in patients with lower sexual desire $(p=0.002)$.

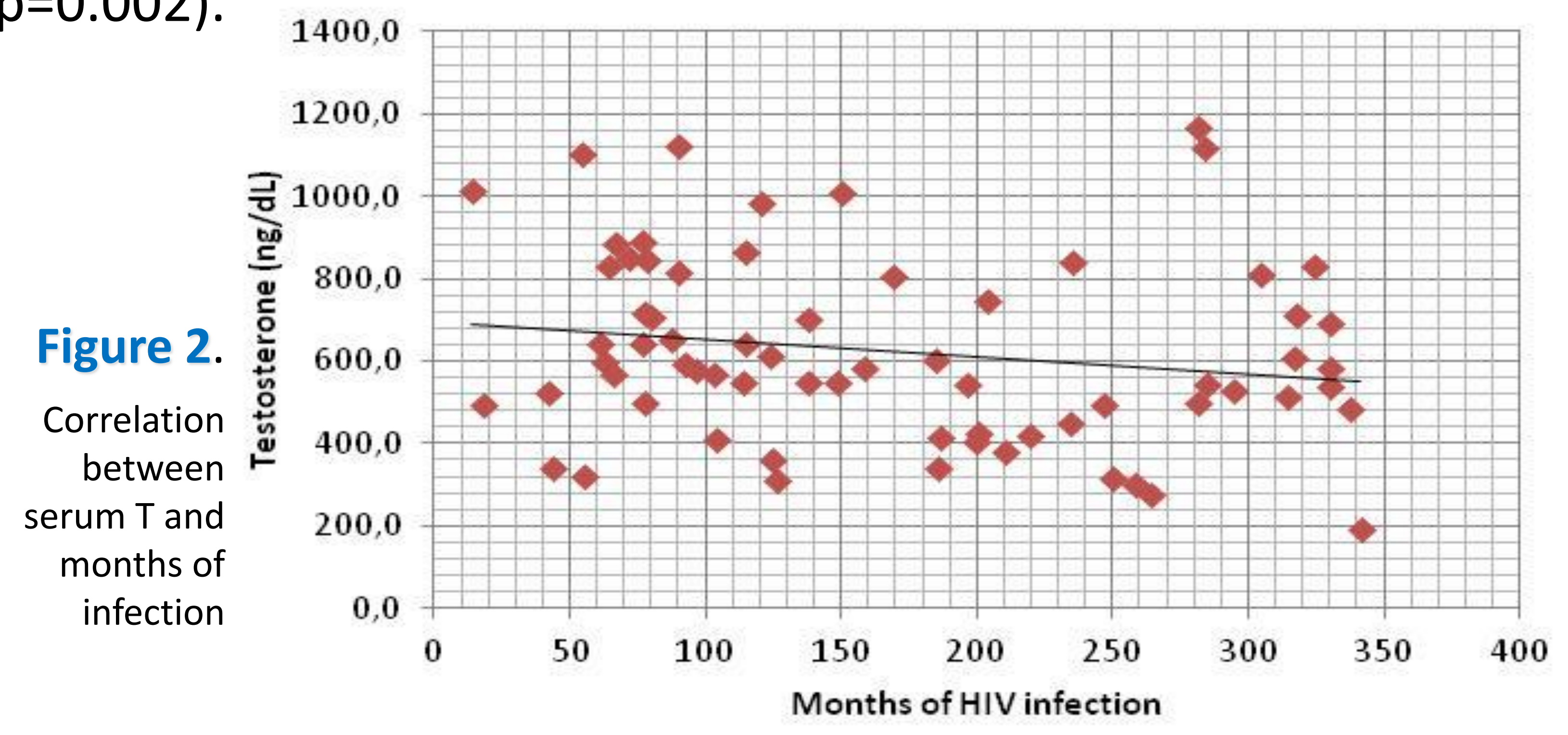

DISCUSSION

The percentage of ED and T deficiency are higher and occurs earlier in HIV-infected men than healthy subjects, supporting the hypothesis of a premature aging of the hypothalamicpituitary-gonadal axis in these patients (3). However, serum $T$ levels seems to be not correlated with IIEF-15 and SIEDY scores, suggesting that ED should not be directly related to the decline of serum $T$ levels. Thus, HIV-infection itself, age-related comorbidities and psycho-emotional status seem to be the strongest risk factors in the development of ED. Furthermore, neither of validated questionnaires seem to be sufficiently trustworthy in the study of sexual function in HIV-infected men, but SIEDY could be more reliable than IIEF-15 in HIV-infected patients with other comorbidities.

\section{REFERENCES}

(1) Samaras K. Best Pract Res Clin Endocrinol Metab. 2011;25(3):vii-viii; (2) Kalyani RR et al. Endocrinol Metab Clin North Am. 2007;36(2):333-348; (3) Rochira V et al. PLoS One. 2011;6(12):e28512; (4) Fanelli et al. Steroids 2011;76(3):244-253; (5) Rosen et al. Urology. 1997:49(6):822-830. (6) Corona et al. J Sex Med. 2012;9(8):2017-2026. (7) Bhasin et al. JCEM 2010;95(6):611-618. 